\title{
DISPOSITIVOS E CONEXÕES DA REDE DE ATENÇÃO PSICOSSOCIAL (RAPS) DE PORTO ALEGRE - RS
}

Gabriela Lemos de Pinho Zanardo

Pontifícia Universidade Católica do Rio Grande do Sul

\section{Desirée Luzardo Cardozo Bianchessi}

Hospital de Clínicas de Porto Alegre

Kátia Bones Rocha

Pontifícia Universidade Católica do Rio Grande do Sul

\begin{abstract}
Resumo
O estudo objetiva conhecer os dispositivos de trabalho que favorecem a funcionalidade da Rede de Atenção Psicossocial, através da perspectiva de trabalhadores de Porto Alegre, e investigar como acontece a articulação entre os diferentes pontos da rede. Trata-se de pesquisa qualitativa, com delineamento exploratório-descritivo. Foram entrevistados treze profissionais da saúde dos diferentes níveis de atenção e as entrevistas foram submetidas à Análise de Conteúdo. Os resultados foram divididos em dois eixos temáticos: (1) dispositivos que auxiliam na formação e funcionalidade da rede e (2) conexão entre os pontos da rede: afastamentos e dificuldades na articulação. A partir do exposto, considera-se que as equipes aderem ao movimento de mudança da lógica de assistência e esforçam-se em estreitar a comunicação entre si, embora existam práticas ainda incipientes. Evidencia-se o fato de que há pouco contato entre os hospitais e demais serviços, sendo este um trabalho a ser desenvolvido nas equipes.
\end{abstract}

Palavras-chave: saúde mental; política de saúde; serviços de saúde pública; intervenção psicossocial.

\section{DEVICES AND CONNECTIONS IN THE PSYCHOSOCIAL ASSISTANCE NETWORK (RAPS) OF PORTO ALEGRE - RS}

\begin{abstract}
The goals of this study are to know the work devices which favor the Psychosocial Assistance Network from the workers' perspective in Porto Alegre, and to investigate how the articulation among different points of the network happens. It is a qualitative research, with an exploratory-descriptive design. We interviewed thirteen health care professionals from several levels of care, and the interviews were analyzed with Content Analysis. We split the results into two thematic axes: (1) devices which help creating and featuring the network, and (2) connection among the network points: estrangement and difficulties in the articulation. From these, we consider that professionals adhere to the movement of changing the assistance logic, and try to narrow communication among themselves, even though some practices are still at the beginning. We highlight the fact that there is little contact between hospitals and other health care services, being this one of the points to be developed in the teams.
\end{abstract}

Keywords: mental health; health policy; public health services; psychosocial intervention. 


\title{
DISPOSITIVOS Y CONEXIONES DE LA RED DE ATENCIÓN PSICOSOCIAL DE PORTO ALEGRE - RS
}

\begin{abstract}
Resumen
El objetivo del estudio fue evaluar los dispositivos de trabajo que favorecen la funcionalidad de la Red de Atención Psicosocial (RAPS) desde la perspectiva de los trabajadores de Porto Alegre; y investigar como lo hace la articulación entre los puntos de la red. Es una investigación cualitativa con un diseño exploratorio descriptivo. Trece trabajadores de los diferentes niveles de atención fueron entrevistados y las entrevistas fueron sometidas a análisis de contenido. Los resultados se dividieron en: (1) dispositivos que ayudan en la formación y funcionalidad de la red y (2) conexión entre los puntos: dificultades en la articulación. Se considera que los equipos están cambiando la lógica de servicio y se esfuerzan para la comunicación más cerca entre sí, aunque todavía hay prácticas incipientes. Es evidente el hecho de que hay muy poco contacto entre los hospitales y otros servicios, que es un trabajo a realizar en equipos.
\end{abstract}

Palabras clave: salud mental; política de salud; servicios de salud pública; intervención psicosocial.

\section{INTRODUÇÃO}

A atual política nacional de saúde mental preconiza um modelo de assistência que visa instituir um atendimento humanizado, com novas práticas de atenção, assegurando a inclusão dos usuários na sociedade, reforçando seus direitos e deveres como cidadãos. Construída a partir do movimento da Reforma Psiquiátrica e oficializada com a promulgação da Lei no 10.126 (Presidência da República, Casa Civil, Subchefia para Assuntos Jurídicos, 2001), que dispõe sobre a proteção e os direitos dos usuários e redireciona o modelo assistencial.

Esse novo modelo propôs a criação de uma rede de serviços substitutiva ao hospital, que após 2011 passa a ser chamada de Rede de Atenção Psicossocial (RAPS) (Ministério da Saúde, 2011/2013), composta por serviços territoriais, dentre eles: Centro de Atenção Psicossocial (CAPS), ambulatório especializado, hospital-dia, equipes de atenção básica, Consultório na Rua, Centro de Convivência, Núcleo de Apoio à Saúde da Família (NASF), Sala de Estabilização, Unidade de Pronto Atendimento (UPA), emergência hospitalar, Unidade de Acolhimento, serviço de atenção em regime residencial, Serviço Residencial Terapêutico (SRT), leitos de psiquiatria em hospital geral e serviço hospitalar de referência, assim como estratégias de reabilitação psicossocial. Além da criação de serviços, a política reforça a importância da formação e capacitação dos profissionais para que proponham novas práticas e atuem no processo de transformação do modelo de cuidado em saúde mental. Evidencia-se a necessidade de mudar a lógica das ações e os modelos de gestão.

Estudos como de Venturini (2010) mostram que ainda é forte a visão hospitalocêntrica e a internação é vista como principal dispositivo de tratamento, encontrando-se uma frequência significativa de reinternações. Nesse sentido, estudos realizados em diferentes regiões do país vêm identificando a ocorrência de reinternações psiquiátricas: no Piauí $55,7 \%$ dos usuários apresentaram 
reinternações no ano de 2004 (Parente et al., 2007), em Ribeirão Preto 34\% das internações entre 2006 e 2007 representavam reinternações (Castro, Furegato \& Santos, 2010) e no Rio Grande do Norte a reincidência de internações ultrapassou 60\% entre 2007 e 2008 (Bezerra \& Dimenstein, 2011).

Com relação as elevadas taxas de reinternação, Bezerra e Dimenstein (2011) evidenciam que o alto número no estado do Rio Grande do Norte pode ter relação direta com a ineficácia da rede de atenção extra hospitalar, assim como a ausência de alguns serviços no período estudado, como CAPS III, assim como leitos em hospitais gerais e Centros de Convivência e Cultura. Além disso, as autoras apontam para a pouca articulação da rede de serviços substitutivos que deem suporte à crise, o que faz com que o hospital psiquiátrico ainda seja o principal local de atendimento. No mesmo sentido, Parente et al. (2007) também referem que os serviços extra hospitalares não estavam sendo instalados na proporção adequada para atender a população. Ademais, os estudos mostram que questões como a falta de esclarecimento aos usuários e famílias sobre a importância do tratamento continuado, o baixo suporte familiar, o uso irregular da medicação e a não continuidade da atenção após a internação, também são apontados como fatores que levam as reinternações (Bezerra \& Dimenstein, 2011; Castro et al., 2010; Parente et al., 2007). A interação desses diferentes fatores pode auxiliar na compreensão das discrepâncias entre as taxas de reinternação.

A manutenção do modelo de cuidado centrado nos hospitais ainda reverbera, como apontado por estudo realizado por Salles e Barros (2007), em que usuários e familiares traziam ainda um discurso manicomial, no qual a internação aparecia como a melhor forma de tratamento e a reinternação seria consequência da suspensão da medicação. Os entrevistados também relataram desconhecer os serviços substitutivos, demonstrando a falta de articulação entre os serviços e de orientação aos usuários e famílias, assim como a ausência de serviços na região. Na mesma direção, Bertagnoni, Marques, Muramoto e Mângia (2012), ao acompanhar itinerários terapêuticos de pessoas com transtornos mentais, constataram que os usuários, que atualmente possuem vínculo com a atenção básica, não possuíam referência de cuidado especializado em saúde mental, exceto pelo serviço hospitalar de urgência e a internação psiquiátrica.

Ramos, Guimarães e Enders (2011) ainda destacam que existe uma relação direta entre a falta de articulação da rede de atenção e o fenômeno das reinternações psiquiátricas, também denominada como porta giratória. No mesmo sentido, outros autores (p. ex., Caçapava \& Colvero, 2008; Chiaverini, 2011) apontam que uma rede bem instrumentalizada (serviços, profissionais e recursos), que permite melhor entendimento do sistema e se preocupa com a dimensão relacional da assistência, é capaz de produzir melhores fluxos, garantir o cuidado continuado e melhorar a resolubilidade da atenção.

Sendo assim, justifica-se a necessidade e relevância de investigar 
questões relacionadas à formação e funcionalidade da RAPS, para que estes conhecimentos reflitam na melhoria da prática assistencial. Para isso estabeleceu-se como objetivos deste estudo: conhecer os dispositivos de trabalho que favorecem a funcionalidade da Rede de Atenção Psicossocial (RAPS), através da perspectiva de trabalhadores de Porto Alegre; assim como investigar como acontece a articulação entre os diferentes pontos da rede.

\section{MÉTODO}

Esta pesquisa consistiu em um estudo qualitativo, com delineamento exploratório-descritivo, realizada com profissionais de seis serviços públicos de saúde de Porto Alegre, sendo dois representantes de cada nível assistencial, conforme descrito a seguir.

\section{Participantes}

Participaram 13 trabalhadores da saúde, representantes de seis serviços públicos de saúde de Porto Alegre, sendo: uma Unidade Básica de Saúde (UBS), uma Unidade de Saúde da Família (USF), um Centro de Atenção Psicossocial (CAPS II), uma Equipe de Saúde Mental (ESM), uma internação de Hospital Psiquiátrico (HP) e uma internação psiquiátrica de Hospital Geral (HG). Devido à importância da heterogeneidade dos participantes, foram entrevistados diferentes profissionais, sendo eles: 2 agentes comunitárias de saúde (ACS), 2 assistentes sociais, 2 enfermeiras, 1 médico de família e comunidade, 1 médico psiquiatra, 1 nutricionista, 2 professores de educação física, 1 psicólogo e 1 técnica de enfermagem. As falas serão identificadas pela categoria profissional e o respectivo serviço de origem, através das siglas descritas anteriormente.

\section{Instrumentos}

Foram utilizadas entrevistas semiestruturadas (Flick, 2004) com as seguintes questões norteadoras: "Fale-me sobre o processo de chegada do usuário com transtornos mentais ao serviço; Como se dá a articulação entre o serviço e outras equipes de saúde?; Fale-me a respeito das atividades desenvolvidas para atenção a esse usuário; Qual a sua opinião a respeito do tratamento oferecido (potencialidades e vulnerabilidades da assistência)? ".

Procedimentos de coleta e análise de dados

Foram contatados os coordenadores destes serviços, que indicaram ao menos dois profissionais de cada equipe para participar do estudo, considerando seu caráter voluntário. As entrevistas foram realizadas nos serviços de saúde, sendo gravadas com autorização dos participantes e posteriormente transcritas e analisadas através da Análise de Conteúdo (Bardin, 2011). 


\section{Procedimentos éticos}

Todos os entrevistados assinaram o Termo de Consentimento Livre e Esclarecido. A pesquisa foi aprovada pelos Comitês de Ética e Pesquisa do hospital proponente (parecer no 258.412), do hospital coparticipante (parecer no 002/2013) e da Secretaria Municipal de Saúde (parecer no 260.720), seguindo a Resolução 466/12 do Conselho Nacional de Saúde (Ministério da Saúde, 2012a).

\section{RESULTADOS E DISCUSSÃO}

Com a instituição da RAPS (Ministério da Saúde, 2011/2013) foram unificados os diferentes serviços que abrangem os cuidados em saúde mental, reforçando o modelo de atenção com base no território. Suas diretrizes visam garantir o acesso e a qualidade dos serviços, ofertando cuidado integral e assistência multiprofissional, sob a lógica interdisciplinar, oferecer atenção humanizada e centrada nas necessidades das pessoas, diversificar as estratégias de cuidado com ênfase em serviços comunitários e organizar os pontos de atenção em rede regionalizada, com estabelecimento de ações intersetoriais.

Considerando estas diretrizes, buscou-se destacar os dispositivos de trabalho descritos pelos profissionais que auxiliam na formação e na funcionalidade da rede, sendo este o primeiro eixo temático apresentado, com suas respectivas categorias de análise. Nessa análise, utilizou-se o conceito de dispositivo proposto por Foucault (2000, p. 244), que diz respeito a um conjunto heterogêneo de "discursos, instituições, organizações arquitetônicas, decisões regulamentares, leis, medidas administrativas, enunciados científicos, proposições filosóficas, morais, filantrópicas". Relaciona-se à rede que pode ser estabelecida entre elementos ditos e não ditos, que exigem uma modificação de funções e surge em determinado momento histórico com objetivo de responder a uma urgência. Na atenção à saúde mental surgem como alternativas ou respostas à exigência de uma nova lógica de cuidado.

Complementando o entendimento sobre a formação da RAPS, apresentase na sequência o segundo eixo temático e suas categorias, denominado: Conexão entre os pontos da rede: afastamentos e dificuldades na articulação.

Dispositivos que auxiliam na formação e na funcionalidade da rede - RAPS

$\mathrm{Na}$ análise das entrevistas, foram identificados dispositivos apontados pelos profissionais que exercem a função de potencializar e fortalecer a RAPS no dia a dia dos serviços. Para sistematizar esses achados, foram estabelecidas as seguintes categorias: apoio matricial, reuniões, contato imediato, identificação da demanda e busca ativa.

O dispositivo do Apoio Matricial, ou Matriciamento foi mencionado por profissionais dos diferentes níveis de atenção, conforme segue: 
"Com as Unidades Básicas existe um serviço de interconsulta e matriciamento, através do qual os casos são discutidos com os médicos de família ou os clínicos gerais [...] no caso das UBS existem reuniões realizadas in loco, dentro das unidades [...] E as ESF da região, que ainda não estão cobertas pelo NASF, funcionam num esquema de interconsulta, que os médicos ou enfermeiros representantes das unidades comparecem a uma reunião mensal onde os casos são discutidos". (Médico Psiquiatra, ESM)

"A gente faz as reuniões de matriciamento com as equipes especializadas e também em relação à rede [...] aí o que acontece: esse paciente passa por uma consulta é levado para matriciamento e no matriciamento a gente analisa e avalia, para que tipo de atendimento vai". (Profissional 12, UBS)

Segundo Campos e Domitti (2007) o Apoio Matricial é uma metodologia de trabalho que tem como objetivo assegurar retaguarda especializada, tanto assistencial quanto suporte técnico-pedagógico, para profissionais ou equipes de referência responsáveis pela atenção a problemas de saúde e condução de casos individuais, familiares ou comunitários. Ela é complementar ao mecanismo de referência e contrarreferência e aos centros de regulação, dependendo da construção compartilhada de diretrizes clínicas e sanitárias entre as equipes de apoio e de referência. Conforme Chiaverini (2011) os serviços passam por um processo de mudança das relações, visando um cuidado colaborativo, através da transformação da lógica tradicional dos sistemas de saúde em ações mais horizontais, que integrem os diferentes profissionais e saberes, nos diferentes níveis assistenciais.

Diversos autores (Alves \& Guljor, 2004; Ceccim \& Ferla, 2006; Cecílio, 2004) problematizam a rigidez e a burocratização das redes de atenção à saúde sistematizada através do princípio de hierarquização dos serviços, assim como o mecanismo de referência e contrarreferência. A hierarquização faz com que os serviços devam adequar-se aos diferentes níveis de atenção, o que dificulta o acesso. Por sua vez o sistema de referência e contrarreferência criou uma assistência repartida, onde não se cria vínculo entre usuários e profissionais, estimulando a prática do encaminhamento (Zambenedetti \& Silva, 2008). Nesse sentido, o apoio matricial pretende personalizar o sistema de referência, estimulando e facilitando o contato direto entre equipes, que pode se dar mediante encontros periódicos com objetivo de discutir casos ou problemas de saúde selecionados pela equipe de referência, elaborar projetos terapêuticos e firmar linhas de intervenção entre os profissionais envolvidos (Campos \& Domitti, 2007).

Os trabalhadores entrevistados ressaltaram o matriciamento como a possibilidade das equipes interagirem em apoio mútuo, reunindo os conhecimentos a respeito de um indivíduo, fazendo o acompanhamento e sendo corresponsáveis pelo cuidado do usuário. Dessa forma, trabalham de acordo com 
a perspectiva proposta pelos autores (Campos \& Domitti, 2007; Chiaverini, 2011) e favorecem a construção conjunta dos projetos terapêuticos.

"Daí a importância dessas discussões com o matriciamento, dos colegas que deem um aporte, da gente poder entre si, no sentido de ajudar, se capacitar para poder atender, qualificar o atendimento". (Médico de Família e Comunidade, UBS)

"[Em caso que necessita de psicoterapia] é agendado com a psicóloga e ele vai manter um atendimento e mantém vínculo conosco". (Assistente Social, UBS)

Apontaram também o avanço e qualificação das relações e da formação da rede através deste dispositivo, embora enfatizassem que esta relação está em construção.

\footnotetext{
"Hoje temos o sistema de matriciamento, que acho que deu uma qualificada. Não é mais aquele encaminhamento em papel que variava a qualidade, não tinha retorno. Referência e contrarreferência, na verdade é um encaminhamento". (Médico de Família e Comunidade, UBS)

"A ideia do matriciamento é boa, mas não dá para ficar restrito a isso. Sou a favor da ideia, acho que ajuda sim [...]. Acontece que tem demandas específicas que aí tu precisas ter serviço substitutivo". (Psicólogo, ESM)
}

Esses achados vão ao encontro de estudos que buscam conhecer e analisar o trabalho dos Núcleos de Apoio a Saúde da Família - NASF - (Ministério da Saúde, 2008; 2011), equipes multiprofissionais criadas para assegurar a retaguarda especializada à atenção básica. Os NASF visam ampliar e qualificar as ações nesse nível de atenção através do apoio matricial às equipes da atenção básica. Como estratégia recente do Ministério da Saúde, implementada a partir de 2008, vem encontrando algumas resistências, conforme constatam as pesquisas de Cela e Oliveira (2015) e Ferro, Silva, Zimmermann, Castanharo e Oliveira (2014). Os autores mostram que alguns profissionais das ESF e UBS estudadas apresentam dificuldades com o novo modelo de trabalho, já que demanda tempo para o encontro e troca com os profissionais do NASF e isso implica a diminuição do número de consultas (Ferro, Silva, Zimmermann, Castanharo, \& Oliveira, 2014). Apontam também uma prática fragmentada, ainda centrada nos atendimentos individuais (principalmente nas consultas médicas) e nos encaminhamentos, numa lógica ambulatorial de cuidado (Cela \& Oliveira, 2015; Ferro et al., 2014), assim como na realização de atendimentos conjuntos entre profissionais do NASF, porém sem a inclusão de profissionais da ESF (Cela \& Oliveira, 2015).

Por outro lado, os estudos também indicam que alguns profissionais reconheciam a importância do apoio matricial e do trabalho interdisciplinar, compreendendo os sujeitos/famílias em sua complexidade e a necessidade de articular saberes para alcançar a integralidade (Cela \& Oliveira, 2015; Ferro et al., 2014). Outros trabalhadores já iniciaram o movimento de atuar sob essas 
diretrizes desde a constituição das equipes do NASF (Cela \& Oliveira, 2015), construindo estratégias através da troca de conhecimentos e experiências das especialidades envolvidas e também questionando as demandas das equipes da ESF, problematizando concepções de saúde cristalizadas e permitindo a revisão de posturas e intervenções (Sundfeld, 2010).

Outro dispositivo apontado como importante para a formação da RAPS e como estratégia para o trabalho conjunto foram as reuniões, sejam elas para a discussão dos planos terapêuticos, para buscar informações, articular diferentes serviços ou mesmo para rever questões da organização e gestão dos serviços. Esse achado está de acordo com Lapischies et al. (2012) que apontam o trabalho em equipe na atenção psicossocial como fator essencial para obter sucesso no cuidado aos usuários, visto que assim é possível ultrapassar as barreiras e saberes de cada profissão, produzindo um conhecimento múltiplo e integrado.

Segundo os entrevistados, as reuniões de equipe apareciam como a forma mais frequente e formal de comunicação, todos os participantes referiram que aconteciam reuniões sistemáticas em seus serviços, semanais ou mensais:

"Tem uma reunião de equipe [...] a gente discute casos específicos, não só dos pacientes, mas da unidade, se a gente encontra alguma dificuldade na assistência, para discutir o que a gente pode fazer para melhorar isso". (Enfermeira A, HG)

As reuniões também ocorrem entre os diferentes serviços visando aproximar, articular e integrar esses nós da rede. Nesse sentido, os achados apontam na mesma direção que Dimenstein et al. (2012) que referem a importância da estruturação de uma rede articulada, que abarque as diferentes necessidades da pessoa com transtorno mental para que se supere o modelo asilar. Os entrevistados mostraram que essa articulação começa a ocorrer através de reuniões sistemáticas, não só da rede de saúde, mas também de serviços intersetoriais, como da assistência social e justiça, por exemplo.

"Tem uma reunião mensal também com os serviços - a gente chama de 'redinha'- de cada microrregião aqui do Bairro. [...] É mais para discussão de caso, para afinar as ações, articular mesmo o trabalho". (Assistente Social, UBS)

"Nessa reunião a gente tem agora uma articulação com os CREAS, CRAS, com Equipe de Saúde Mental, Equipe de Matriciamento. A gente conhece as pessoas, discute os casos, os encaminhamentos". (Professora de Educação Física, CAPS II)

Além das reuniões, foi apontado como de significativa importância o trabalho e a articulação realizados através de um contato mais direto e informal, traduzido como contato imediato. Os profissionais referiram que a comunicação entre eles também acontecia fora dos momentos formais de reunião. Dentro dos serviços há a troca diária entre os profissionais do turno, que estão em contato direto com os usuários ou em atividade específica: 
"Acho que já é um avanço a gente participar dos rounds, discutir junto sobre o paciente [...] e fora que a gente discute toda a hora, a gente está sempre avaliando o paciente". (Enfermeira B, HG)

Já entre os diferentes serviços, os entrevistados relataram que o contato acontecia devido à proximidade e parceria estabelecida entre eles, seja por terem trabalhado juntos em outro momento ou pelo contato estabelecido inicialmente nas reuniões.

"Tem esse contato mais do dia a dia que a gente consegue fazer nesse momento, por uma construção de trabalho que a gente fez. [...] Então manda um encaminhamento por escrito para uma pessoa no outro serviço, mas já articulou isso por telefone, já vai chegar lá e vai ter um acolhimento". (Assistente Social, UBS)

Através da comunicação dos profissionais, seja dentro ou entre serviços, é possível realizar a identificação da demanda dos usuários. Entender as demandas e necessidades deles, segundo autores como Campos e Domitti (2007) e Dimenstein et al. (2012), é fundamental para que as equipes pensem e formulem seus projetos terapêuticos e a intervenção que melhor irá atendê-los. Conforme esses autores, quando bem avaliados e encaminhados, os usuários tendem a ter melhor aderência e motivação no tratamento, além de não sobrecarregarem as equipes especializadas com atendimentos que podem ser realizados em um serviço de menor complexidade.

"Nós dificilmente recebemos pacientes sem ter discutido com a atenção básica [...] a gente recebe os pacientes que são já um pouco, eu vou usar o termo 'triados' pela atenção básica, aqueles que mais preocupam eles e muitas vezes os mais graves, pacientes de difícil manejo". (Psicólogo, ESM)

Barros, Tung e Mari (2010) compreendem que a participação efetiva da atenção primária na RAPS gera uma menor sobrecarga na rede, principalmente através da distribuição dos casos conforme sua gravidade nos serviços de saúde que a compõe. A instrumentalização desses serviços pode evitar o agravamento dos casos, o que influenciaria diretamente os Serviços de Emergência Psiquiátrica, no sentido de diminuir internações desnecessárias, permitindo o acesso de casos mais graves à atenção secundária. Na mesma direção, Rocha et al. (2013) mostram que quanto maior a integração entre atenção primária e especializada em saúde mental, maior é a possibilidade de acesso aos serviços de saúde. Por isso, Dimenstein et al. (2012) destacam a importância do apoio matricial para fortalecer a organização da RAPS, pois assim os profissionais podem contribuir na melhoria da capacidade resolutiva das equipes de referência, qualificando esses trabalhadores para uma atenção mais ampliada e integral.

"Depende se é um paciente que está controlado, com orientação, com a medicação, o médico avalia se ele pode ser acompanhado aqui [...]" (Técnica de Enfermagem, USF)

A fala da profissional acima vem ao encontro do que é proposto nos 
Cadernos de Atenção Básica (Ministério da Saúde, 2013), que enfatizam que o cuidado em saúde mental na atenção primária deve ser aprimorado, com intervenções que levem em consideração a singularidade e subjetividade de cada usuário para garantir um cuidado integral à saúde. Além disso, cabe à atenção primária identificar riscos, necessidades e demandas de saúde, criando intervenções efetivas, assim como acompanhar e organizar o fluxo dos usuários entre os pontos da rede.

O último dispositivo referido pelos entrevistados foi a busca ativa. Segundo os trabalhadores, a busca ativa permite que seja realizado o acompanhamento contínuo dos usuários em seu território, entendendo suas demandas e necessidades. Por meio do contato com a comunidade, os Agentes Comunitários de Saúde (ACS) fazem um papel relevante nesse sentido, auxiliando na descoberta de novos casos e acompanhamento de usuários com transtornos mentais.

Em consonância com esses achados, Caçapava e Colvero (2008) apontam a importância e responsabilidade atribuída aos ACS no cuidado a usuários em sofrimento psíquico. Conforme os dados encontrados na presente pesquisa, a possibilidade de acompanhar os usuários e poder realizar sua busca ativa quando se encontram faltosos ou impossibilitados de comparecer aos serviços, além de monitorar a situação de saúde e a adesão ao tratamento (medicamentoso ou atendimentos), faz do ACS ferramenta fundamental no cuidado em saúde mental, assim como destacado pelos autores (Caçapava \& Colvero, 2008). Esse fato fica evidenciado no relato abaixo:

"A pessoa tem alta do âmbito hospitalar, muitas vezes não volta, tu não ficas nem sabendo se aquela pessoa está bem ou não. E a diferença do posto é essa, como tem o ACS, tem a visita domiciliar. Tu ficas sabendo se a pessoa está bem, se está aderindo, aí tu fazes a busca ativa". (Agente Comunitária de Saúde, USF)

Segundo os Cadernos de Atenção Básica (Ministério da Saúde, 2013) esse cuidado na atenção primária é considerado estratégico, pois facilita o acesso das equipes aos usuários e vice-versa. As ações são desenvolvidas em um espaço conhecido, próximo à vida das pessoas, com o mais alto grau de descentralização e capilaridade. Nesse sentido, os profissionais da atenção primária entrevistados reconheciam-se enquanto responsáveis pelo cuidado continuado dos usuários, mesmo que eventualmente fossem atendidos em outro serviço (como na internação) e relataram a importância desse acompanhamento, do monitoramento em relação à adesão ao tratamento.

"(Quem fica responsável pelo usuário?) O profissional que fez o acolhimento e o serviço de atenção básica, porque depois ele volta para nós e quem é responsável para ver se a coisa está andando, se não está andando, se foi, se não foi... é a atenção básica". (Assistente Social, UBS) 


\begin{abstract}
"Esse sistema de renovação de receita, muitas vezes, nos permite ver problemas. A gente começa a renovar e vê que passou muito tempo e aquela pessoa não está consultando. Muitos desses nós temos colocado no sistema de visita para ver o que está acontecendo. Aí vai lá e tem-se encontrado casos graves e que estavam sem atendimento porque a pessoa não sai de casa, e aí nós vamos passar a ver em casa". (Médica da família e comunidade, UBS)
\end{abstract}

Segundo a Política Nacional de Atenção Básica (Ministério da Saúde, 2012b), a Saúde da Família é destacada como principal estratégia para expansão e consolidação da atenção primária. Com território adstrito, composta por equipe multiprofissional e ACS, fica evidente a diferença da composição das UBS e USF, conforme destacado na fala da seguinte profissional:

"Em termos de estrutura numérica de profissionais, a modificação do serviço e adequação à estrutura prevista, que atualmente é ser Estratégia de Saúde da Família. Então se tudo for transformado em ESF, melhor. Porque a gente ter população cadastrada, estrutura para atender essa população e Agente Comunitário, bom, daí melhora". (Assistente Social, UBS)

Nesse sentido, Porto Alegre atualmente conta com uma cobertura de $65,7 \%$ da atenção básica, sendo a cobertura efetuada por Estratégias de Saúde da Família estimada em 53,6\%. Já o número de Agentes Comunitários de Saúde em 2017 corresponde a 765 agentes, que cobririam cerca de 29,7\% do território (Prefeitura Municipal de Porto Alegre, 2017). Conforme os dados e as falas apontadas acima, a cidade ainda precisa adequar as Unidades Básicas de Saúde para transformarem-se em Estratégias de Saúde da Família, implementando também o Programa de Agentes Comunitários de Saúde, que alcança uma pequena parcela das comunidades e da população atendida pelos serviços. Porém, ainda que com infraestrutura em defasagem para uma cobertura adequada da população, entende-se que a busca ativa, assim como apontada pelos profissionais entrevistados, é uma importante ferramenta de cuidado para saúde.

Cabe destacar que o termo busca ativa foi originalmente usado para designar uma ação em vigilância epidemiológica e sanitária com fim de ir à procura de indivíduos e identificar precocemente casos suspeitos (doenças e agravos de notificação compulsória) e confirmar rapidamente para que se fizessem as orientações das medidas de controle (Ministério da Saúde, 2005). Porém, houve uma transformação do sentido do termo a partir da utilização de alguns princípios do SUS e da reforma psiquiátrica como a integralidade e a desinstitucionalização. Segundo Lemke e Silva (2010, p. 285) busca ativa "também passou a ser entendida como um movimento de ir à contracorrente do automatismo da demanda espontânea, no sentido de cartografar as necessidades de saúde para além dos agravos de notificação compulsória de determinado território".

Através da proposta de trabalhar com os sujeitos em seu território, 
transformou-se o termo busca ativa em uma postura política de trabalho no território (Lemke \& Silva, 2010), incluindo a dimensão da subjetividade nas práticas de saúde. Ou seja, as intervenções ultrapassam o caráter individual e biológico e passam a entender os sujeitos em seu contexto de vida. Atualmente, espera-se que a busca ativa faça parte das práticas em saúde, visto que já é considerada uma atribuição de todos os profissionais da ESF (Ministério da Saúde, 2012b).

\section{Conexão entre os pontos da rede: afastamentos e dificuldades na articulação}

Para que os serviços de saúde funcionem como uma rede, os diferentes níveis de atenção devem trabalhar de forma horizontal e integrada, trocando informações e responsabilidades. Chiaverini (2011, p. 203) refere que "trabalhar em rede é tecer possibilidades, aumentando as oportunidades de atuação dos indivíduos, dos profissionais e dos dispositivos de saúde numa crescente corrente de corresponsabilidade". E para que isso aconteça os pontos da rede devem estar conectados. Dentro deste eixo são analisadas as categorias: afastamento do hospital, continuidade $x$ rupturas do cuidado e regulação de leitos.

O afastamento do hospital foi identificado pelos diferentes trabalhadores entrevistados, caracterizado pelo contato raro entre atenção básica e/ou serviços especializados e unidades de internação psiquiátrica, que ocorreu em casos pontuais.

\footnotetext{
"A gente fez alguns trabalhos com o Hospital B (Hospital Geral) e foi possível ter uma proximidade, mas pontual relativa ao paciente". (Psicólogo, ESM)
"Eles saem com uma nota de alta. Algumas vezes eles entram em contato. Uma vez uma Assistente Social me ligou que tinha uma usuária minha internada lá [...] Mas foi o único hospital (Hospital Geral) que me deu retorno". (Agente Comunitária de Saúde, USF)
"A gente não tem essa proximidade e essa troca com internações, então é uma lacuna". (Professora de Educação Física, CAPS II)

Os profissionais percebiam o afastamento do hospital, referindo que a rede tem uma falha em relação ao nível terciário e não identificavam um movimento deste em iniciar o contato com demais serviços. Esses relatos estão de acordo com estudos como de Campos et al. (2009) que apontam os entraves na formação de parceria entre serviços, assim como a ausência de acompanhamento dos usuários quando internados. Parece que o hospital ainda não superou totalmente o modelo da instituição isolada, de reclusão e estudo das doenças (Foucault, 2000; Lancetti \& Amarante, 2012), apesar dos esforços e do movimento para essa nova configuração em rede.

"A rede com as internações não é uma rede muito... é clivada, existe rede básica, serviços substitutivos em geral - inclui o CAPS nisso - e internação. Quando passa para o hospital, a minha impressão é que o paciente fica muito pensado só ali, por aquele profissional. Ele é o 
responsável, a equipe ali é responsável, eu entendo isso. Mas não vejo que os hospitais queiram fazer rede". (Psicólogo, ESM)

Profissionais dos diferentes serviços apontaram a necessidade de que essa relação melhore. Novas propostas vinham surgindo, ainda que timidamente, para que o hospital se articulasse com os demais serviços da RAPS. Nesse sentido, os profissionais do hospital mostraram que em alguns casos era realizado o contato com outros serviços ainda na internação, conforme as falas a seguir:

\begin{abstract}
"Eu sinto falta de fazer uma contrarreferência. Eu sei que para a emergência, da onde ele veio, não vou encaminhar de volta, mas à atenção básica. A gente tem aqui no serviço um projeto dos residentes, mas não são todos que fazem parte desse projeto, da gente poder conversar se vai pra UBS ou pra ESF, ou para um CAPS mesmo. Eu sinto falta de fazer isso". (Enfermeira B, HG)
\end{abstract}

"Muitas vezes fica a preocupação do pós-alta, como é que ele vai aderir ao medicamento, vai seguir um tipo de acompanhamento [...], em casos mais críticos a gente acaba contatando o médico do CAPS ou Posto de Saúde [...] Mas a gente sabe que isso é bem difícil, que na realidade nem sempre é feito". (Enfermeira A, HG)

Nessa direção, Dimenstein e Liberato (2009) apontam que uma das ações mais urgentes na Saúde Mental é a alta planejada e assistida. Argumentam ser imprescindível ao avanço da reforma psiquiátrica a constituição da rede integrada de serviços substitutivos - serviços de saúde e demais dispositivos presentes na comunidade - porém, é uma das maiores fragilidades, visto que ainda não dispomos de uma rede ágil e resolutiva. As autoras destacam a identificação de um maior número de serviços que trabalham isolados, fechados em suas rotinas, sem comunicação com outros, o que vem ao encontro das falas dos participantes em relação aos hospitais.

Como visto anteriormente, vários estudos associam a diminuição das internações psiquiátricas e a prestação de cuidado continuado à resolutividade da articulação da rede de serviços, ou seja, a funcionalidade da RAPS. Em pesquisa a respeito do efeito da porta giratória, Ramos e Guimarães (2013) mostram que com a criação de serviços e ampliação da RAPS, foi evidenciada uma redução de $6,57 \%$ no número de reinternações do hospital estudado em um período de quatro anos e meio. Outros estudos apontam que há uma relação direta entre o fenômeno da porta giratória e a falta de articulação da RAPS (Bezerra \& Dimenstein, 2011; Dimenstein \& Liberato, 2009; Ramos, Guimarães \& Enders, 2011). Nesse sentido, os dados que surgiram nas entrevistas foram traduzidos na categoria: continuidade $x$ rupturas do cuidado.

"Se ele conseguir se vincular a esse serviço e ele tiver o acompanhamento de uma equipe especializada, ele vai evitar a reinternação". (Assistente Social, HP)

"Teve casos que a gente teve sucesso em começar a afastar um período de internação do outro, aumentar esse período fora do hospital [...] Participando do CAPS do bairro dele, pegando a medicação no posto". (Professor de Educação Física, HP) 
Além disso, os profissionais associaram os sujeitos que precisam de cuidados intensivos na internação a casos de maior gravidade ou que necessitam de atenção de maior complexidade. Por isso, casos assim avaliados acabam exigindo das equipes maior atenção ou atendimento diferenciado.

"Tiveram seis internações psiquiátricas na minha área, são casos bem graves. Então esses, eu não digo que têm prioridade, porque todos têm prioridade, mas esses eu acompanho mais de perto porque é paciente mais de risco, se o paciente chega a internar é porque não está bem". (Agente Comunitária de Saúde, USF)

\begin{abstract}
"Se o paciente é egresso de alguma internação psiquiátrica e ele pertence a região de abrangência da nossa equipe é agendada uma avaliação, sem ele precisar passar pela rede básica, pois geralmente se detecta a necessidade da continuidade do tratamento médico [...] a gente consegue rastrear esses pacientes, inclusive a gente tem um programa continuado de busca ativa desses pacientes". (Médico Psiquiatra, ESM)
\end{abstract}

Por fim, a última categoria analisada nesta temática foi a regulação de leitos. Essa acontece hoje através de uma Central de Leitos que distribui os casos que foram avaliados nas Emergências Psiquiátricas e aguardam em observação para transferência aos leitos disponíveis em unidades de internação de hospitais - gerais ou psiquiátricos.

Com relação ao atendimento à crise, Dimenstein et al. (2012) apontam que esse é um dos problemas mais notórios e de difícil manejo no campo da saúde mental e relatam a preocupação de diversos países em elaborar propostas de intervenção. Consideram que o manejo rápido é decisivo para uma resposta eficaz, de forma a evitar hospitalizações, sofrimento e cronificação. Nesse sentido, Barros et al. (2010) colocam que a agilidade no manejo pode ser limitante se levado em conta a formação dos profissionais para esse atendimento e a efetividade na atenção. Serviços de emergência em geral possuem poucos leitos para observação e acompanhamento da evolução do quadro, o que pode levar a uma decisão precoce de indicação à internação. Por outro lado, os serviços extra hospitalares por vezes não oferecem a mesma estrutura e agilidade encontrada nas emergências.

Em recente revisão de literatura (Barros, Tung \& Mari, 2010) encontrou-se que os Serviços de Emergência Psiquiátrica passaram a realizar uma função central na organização da RAPS, visto que atuam como principal porta de entrada à rede e organizam o fluxo das internações, contribuindo para a redução de internações desnecessárias, além de possibilitarem melhor comunicação entre os diferentes serviços. Porém, na direção contrária aos achados da literatura, as entrevistas apontaram para o fato de que esta talvez não seja a melhor forma de regulação das internações, conforme exemplificam as falas:

"Muitas vezes precisa encaminhar paciente para uma internação, que é feito através da Emergência [...] O que dificulta, às vezes, esse processo é o fato de que, no nosso município, não há possibilidade da 
gente interagir diretamente com a regulação de leitos para reservar vaga. Eventualmente se é identificado que o paciente precisa de internação, ou até quando precisa de algum tipo de tratamento específico [...] de poder referenciar para hospitais que efetivamente possam disponibilizar o tratamento". (Médico Psiquiatra, ESM)

"A internação é via o serviço de Emergência [...] então, quando a gente acha que precisa internar, se encaminha para esse serviço [...] nós não podemos regular, quem regula é só lá na Emergência, não sei se é o melhor lugar para regular isso, ou pelo menos isso ser exclusivo". (Médico da Família e Comunidade, UBS)

Os profissionais ainda questionam sobre os critérios e a qualidade da avaliação realizada na Emergência Psiquiátrica, se a mesma não é um fator que acarreta algumas reinternações, como se percebe na fala abaixo:

"Nos últimos tempos eu tenho observado muitos pacientes reinternando, de tal forma que desvaloriza a internação. São pacientes que não estão, no momento, apresentando riscos. Mas até acho que é uma dificuldade, não sei, se nas Emergências Psiquiátricas, de fazer uma avaliação mais criteriosa, ou dos médicos plantonistas do hospital. [...] É mais uma questão social que poderia ser trabalhada, do que em si uma emergência psiquiátrica". (Enfermeira A, HG)

Os entrevistados também apontam a inexistência de relação com as Emergências Psiquiátricas. Em geral, os usuários são atendidos quando buscam espontaneamente o serviço e não há diálogo com os profissionais que já realizam seu acompanhamento, o que vai na contramão do movimento que se faz quanto à comunicação e integração da RAPS. Isso faz com que esses profissionais que realizam a avaliação conheçam os sujeitos pontualmente, de modo transversal, que pode incorrer no erro de uma internação desnecessária ou da não internação quando há risco já avaliado por outro profissional.

\section{CONSIDERAÇÕES FINAIS}

Embora o processo da reforma psiquiátrica brasileira tenha uma trajetória de aproximadamente quarenta anos, as transformações no campo da atenção à saúde mental continuam acontecendo. Avança-se em relação à concepção dos serviços substitutivos enquanto responsáveis do atendimento e acompanhamento dos usuários com transtornos mentais, para a ideia de que é necessária uma rede com diferentes pontos de atenção, sejam serviços de saúde (em seus diferentes níveis e complexidades de atenção), sejam serviços da assistência, justiça, educação e mesmo as instituições família e comunidade.

Os resultados do presente estudo apontam que as equipes vêm aderindo ao movimento de mudança da lógica de assistência e de relação entre os serviços, esforçando-se em iniciar ou estreitar a comunicação e a relação entre si, ainda que sejam incipientes. Procurou-se destacar os dispositivos que auxiliam nesta articulação e colocam a rede e os sujeitos em movimento. 
Encontrou-se no Apoio Matricial, nas reuniões de rede e outras formas de comunicação das equipes, potentes ferramentas para a integração da mesma.

Evidencia-se o fato de que há pouco contato entre os hospitais e demais serviços, sejam da rede básica, serviços matriciadores ou CAPS, sendo este um trabalho a ser desenvolvido principalmente com as equipes das internações, que já começam a se dar conta da importância do acompanhamento continuado, principalmente devido ao aumento do número de reinternações. Além disso, surge um questionamento quanto à avaliação da necessidade de internação e a regulação dos leitos, hoje exercida pelos Serviços de Emergência Psiquiátrica, outro ponto que aparece conectado de forma bastante frágil em relação aos demais serviços.

Os profissionais destacaram a importância de ter parcerias e uma relação de apoio mútuo com colegas e serviços. Reconheceram a grande demanda de cuidados em saúde mental e a falta de recursos humanos, estruturais e materiais para lidar com ela. Reforçaram a necessidade de investimento na ampliação dos serviços, no aumento do número de profissionais e na sua capacitação, principalmente da atenção primária à saúde, que nem sempre se sentem preparados ou seguros para atender a demanda de saúde mental. Ações técnicopolíticas a nível federal podem ser mais difíceis de serem estabelecidas ou modificadas. Aposta-se em ações locais e regionais, intra e interserviços, como os dispositivos identificados como aqueles que vêm auxiliando na formação e na funcionalidade da rede. Dessa forma, potencializando a capacidade e poder de ação dos sujeitos envolvidos, sejam eles usuários ou profissionais do SUS.

Este estudo apresenta como uma de suas limitações a falta da perspectiva de profissionais dos Serviços de Emergência Psiquiátrica da cidade, apontado como pouco articulado à rede, o que indica maior necessidade de investigação nesse sentido. Apesar dos profissionais entrevistados não serem representativos da rede na sua totalidade, a possibilidade de realizar investigação junto a pessoas com diferentes níveis de formação e profissão, além de serem representantes de diferentes serviços, é um dos pontos fortes desse estudo, apresentando diferentes perspectivas e realidades.

Recomenda-se, conforme os achados desse estudo, que os dispositivos aqui identificados possam ser potencializados para ampliar a funcionalidade da Rede de Atenção Psicossocial, qualificando o cuidado às pessoas com transtornos mentais. No mesmo sentido, futuros estudos podem buscar ampliar essa compreensão a respeito da rede, investigando e identificando outros possíveis dispositivos que promovem a articulação da RAPS, assim como fatores que dificultem a conexão dos serviços. 


\section{DECLARAÇÃO DE CONFLITOS DE INTERESSE}

Não há conflitos de interesse.

\section{REFERÊNCIAS}

Alves, D.S, \& Guljor, A. P. (2004). O cuidado em saúde mental. In R. Pinheiro, \& R. A. Mattos (Eds.). Cuidado: As fronteiras da integralidade (pp. 221-240). Rio de Janeiro, RJ: Hucitec.

Bardin, L. (2004). Análise de Conteúdo. Lisboa: Edições 70.

Barros, R. E. M., Tung, T. C., \& Mari, J. J. (2010). Serviços de emergência psiquiátrica e suas relações com a rede de saúde mental Brasileira. Revista Brasileira de Psiquiatria, 32(Supl. 2), S71-S77. doi:10.1590/S151644462010000600003

Bertagnoni, L., Marques, A. L. M., Muramoto, M. T., \& Mângia, E. F. (2013). Núcleo de Apoio à Saúde da Família e Saúde Mental: Itinerários terapêuticos de usuários acompanhados em duas Unidades Básicas de Saúde. Revista de Terapia Ocupacional da Universidade de São Paulo, 23(2), 153-162. doi:10.11606/issn.2238-6149.v23i2p153-162

Bezerra, C. G., \& Dimenstein, M. (2011). O fenômeno da reinternação: Um desafio à Reforma Psiquiátrica. Mental, 9(16), 303-326. Recuperado de http://pepsic.bvsalud.org/pdf/mental/v9n16/a07v9n16.pdf.

Caçapava, J. R., \& Colvero, L. A. (2008) Estratégias de atendimento em saúde mental nas Unidades Básicas de Saúde. Revista Gaúcha de Enfermagem, 29(4), 573-580.

Recuperado

de http://seer.ufrgs.br/RevistaGauchadeEnfermagem/article/view/7628/4683.

Campos, G. W. S., \& Domitti, A. C. (2007). Apoio matricial e equipe de referência: Uma metodologia para gestão de trabalho interdisciplinar em saúde. Cadernos de Saúde Pública, 3(2), 399-407. doi:10.1590/S0102311 X2007000200016.

Campos, R. T. O., Furtado, J. P., Passos, E., Ferrer, A. L., Miranda, L., \& da Gama, C. A. P. (2009) Avaliação da rede de centros de atenção psicossocial: Entre a saúde coletiva e a saúde mental. Revista de Saúde Pública, 43(Suppl. 1), 16-22. doi:10.1590/S0034-89102009000800004

Castro, S. A., Furegato, A. R. F., \& Santos, J. L. F. (2010). Características sociodemográficas e clínicas em reinternações psiquiátricas. Revista LatinoAmericana de Enfermagem, 18(4), [09 telas]. Recuperado de http://www.scielo.br/pdf/rlae/v18n4/pt_20.pdf. 
Ceccim, R. B., \& Ferla, A. A. (2006). Linha de cuidado: A imagem da mandala na gestão em rede de práticas cuidadoras para uma outra educação dos profissionais de saúde. In R. Pinheiro, \& R. A. Mattos (Eds.). Gestão em redes: práticas de avaliação, formação e participação na saúde ( $p p$. 165184). Rio de Janeiro, RJ: Editora da Abrasco.

Cecílio, L. C. O. (2004) Modelos tecno-assistenciais em saúde: Da pirâmide ao círculo, uma possibilidade a ser explorada. In Ministério da Saúde. VER-SUS Brasil: caderno de textos. (pp. 90-106). Brasília, DF: Ministério da Saúde.

Cela, M., \& Oliveira, I. F. de (2015). O psicólogo no Núcleo de Apoio à Saúde da Família: articulação de saberes e ações. Estudos de Psicologia, 20(1), 3139. doi:10.5935/1678-4669.20150005

Chiaverini, D. H. (Ed.), Gonçalves, D. A., Ballester, D., Tófoli, L. F., Chazan, L. F., Almeida, N., \& Fortes, S. (2011). Guia prático de matriciamento em saúde mental. Brasília, DF: Ministério da Saúde, Centro de Estudo e Pesquisa em Saúde Coletiva. Recuperado de http://bvsms.saude.gov.br/bvs/publicacoes/guia_pratico_matriciamento_sa udemental.pdf

Dimenstein, M., \& Liberato, M. (2009). Desinstitucionalizar é ultrapassar fronteiras sanitárias: O desafio da intersetorialidade e do trabalho em rede. Cadernos Brasileiros de Saúde Mental, 1(1), 1-10. Recuperado de http://stat.intraducoes.incubadora.ufsc.br/index.php/cbsm/article/viewFile/ $1016 / 1142$.

Dimenstein, M., Amorim, A. K. A., Leite, J., Siqueira, K., Gruska, V., Vieira, C., ... \& Bezerril, M. C. (2012). O atendimento da crise nos diversos componentes da rede de atenção psicossocial em Natal/RN. Polis e Psique, Número Temático(2), 95-127. https://doi.org/10.22456/2238-152X.40323

Ferro, L. F., Silva, E. C., Zimmermann, A. B., Castanharo, R. C. T. \& Oliveira, F. R. L. de. (2014). Interdisciplinaridade e intersetorialidade na Estratégia Saúde da Família e no Núcleo de Apoio à Saúde da Família: Potencialidades e desafios. O Mundo da Saúde, 38(21), 129-138. doi: 10.15343/01047809.20143802129138

Flick, U. (2004). Uma introdução à pesquisa qualitativa. (2. Ed.). Porto Alegre, RS: Bookman.

Foucault, M. (2000). Microfísica do Poder. (15. Ed.) Rio de janeiro, RJ: Edições Graal.

Lancetti, A., \& Amarante, P. (2006). Saúde Mental e Saúde Coletiva. In G. W. S. Campos, M. C. S. Minayo, M. Akerman, M. Drumond Júnior, Y. M. Carvalho (Eds.). Tratado de Saúde Coletiva (pp. 615-634). Rio de Janeiro, RJ: Fiocruz; São Paulo, SP: Hucitec. 
Lapischies, S. R. C., Lima, Z. G., Jardim, V. M. R., Coimbra, V. C. C., \& Kantorski, L. P. (2012) O trabalho em serviços da rede de atenção psicossocial: Dificuldades enfrentadas pelos trabalhadores. Cogitare Enfermagem, 17(4), 697-702. doi:10.5380/ce.v17i4.30376

Lemke, R. A., \& Silva, R. A. N. (2010). A busca ativa como princípio político das práticas de cuidado no território. Estudos e Pesquisas em Psicologia, 10(1), 281-295.

Recuperado

de http://www.revispsi.uerj.br/v10n1/artigos/pdf/v10n1a18.pdf.

Ministério da Saúde. (2005). Secretaria de Vigilância em Saúde. Guia de vigilância epidemiológica. 6a edição. Brasília, DF: Ministério da Saúde. Secretaria de Vigilância em Saúde. Recuperado de http://bvsms.saude.gov.br/bvs/publicacoes/Guia_Vig_Epid_novo2.pdf

Ministério da Saúde. (2008). Gabinete do Ministro. Portaria no 154 de 24 de janeiro de 2008. Cria os Núcleos de Apoio à Saúde da Família - NASF. Brasília, DF: Ministério da Saúde. Recuperado de http://bvsms.saude.gov.br/bvs/saudelegis/gm/2008/prt0154_24_01_2008. html

Ministério da Saúde. (2011). Gabinete do Ministro. Portaria no 2.448 de 21 de outubro de 2011. Aprova a Política Nacional de Atenção Básica, estabelecendo a revisão de diretrizes e normas para a organização da Atenção Básica, para a Estratégia Saúde da Família (ESF) e o Programa de Agentes Comunitários de Saúde (PACS). Brasília, DF: Ministério da Saúde. Recuperado http://bvsms.saude.gov.br/bvs/saudelegis/gm/2011/prt2488_21_10_2011. html

Ministério da Saúde. (2011/2013). Gabinete do Ministro. Portaria no 3.088, de 23 de dezembro de 2011 (Republicada em 2013). Institui a Rede de Atenção Psicossocial para pessoas com sofrimento ou transtorno mental e com necessidades decorrentes do uso de crack, álcool e outras drogas, no âmbito do Sistema Único de Saúde. Diário Oficial da União: Brasília, DF. Recuperado http://bvsms.saude.gov.br/bvs/saudelegis/gm/2011/prt3088_23_12_2011_ rep.html

Ministério da Saúde (2012a). Conselho Nacional de Saúde. Resolução nº 466, de 12 de dezembro de 2012. Diretrizes e normas regulamentadoras de pesquisas envolvendo seres humanos. Diário Oficial da União: Brasília, DF. Recuperado

de http://bvsms.saude.gov.br/bvs/saudelegis/cns/2013/res0466_12_12_2012. html 
Ministério da Saúde. (2012b). Secretaria de Atenção à Saúde, Departamento de Atenção Básica. Política Nacional de Atenção Básica. Brasília, DF: Ministério da Saúde. Recuperado de http://189.28.128.100/dab/docs/publicacoes/geral/pnab.pdf

Ministério da Saúde. (2013). Secretaria de Atenção à Saúde, Departamento de Atenção Básica. Cadernos de Atenção Básica: Saúde Mental - 34. Brasília, DF: Ministério da Saúde. Recuperado de http://189.28.128.100/dab/docs/portaldab/publicacoes/caderno_34.pdf

Parente, C. J. S., Mendes, L. P. F., Souza, C. N. S., Silva, D. K. M., Silva, J. C., Parente, A. C. B. V., \& Parente, A. C. M. (2007). O fenômeno de revolving door em hospitais psiquiátricos de uma capital do nordeste brasileiro. Revista Mineira de Enfermagem, 11(4), 381-386. Recuperado de http://www.reme.org.br/content/imagebank/pdf/v11n4a06.pdf.

Prefeitura Municipal de Porto Alegre. (2017). Secretaria Municipal de Saúde. Relatório de Gestão do 10 Quadrimestre de 2017. Secretaria Municipal de Saúde: Prefeitura Municipal de Porto Alegre. Recuperado de http://lproweb.procempa.com.br/pmpa/prefpoa/sms/usu_doc/relatorio_ges tao1quadrimestre_2017.pdf

Presidência da República, Casa Civil, Subchefia para Assuntos Jurídicos. (2001). Lei no 10.216, de 6 de abril de 2001. Dispõe sobre a proteção e os direitos das pessoas portadoras de transtornos mentais e redireciona o modelo assistencial em saúde mental. Diário Oficial da União: Brasília, DF. Recuperado de

http://www.planalto.gov.br/ccivil_03/leis/leis_2001/l10216.htm

Ramos, D. K. R., Guimarães, J., \& Enders, B. C. (2011). Análise contextual de reinternações frequentes de portador de transtorno mental. Interface Comunicação, Saúde, Educação, 15(37), 519-527. http://dx.doi.org/10.1590/S1414-32832011005000015

Ramos, D. K. R, \& Guimarães, J. (2013). Novos serviços de saúde mental e o fenômeno da porta giratória no Rio Grande do Norte. Revista Mineira de Enfermagem, 17(2), 440-445. doi:10.5935/1415-2762.20130033

Rocha, K. B., Pérez, K., Rodríguez-Sanz, M., Alonso, J., Muntaner, C., \& Borrell, C. (2013). Inequalities in the Use of Services Provided by Psychiatrists in Spain: A Multilevel Study. Psychiatric Services, 64(9), 901-907. doi: 10.1176/appi.ps.201100419.

Sundfeld, A. C. (2010). Clínica ampliada na atenção básica e processos de subjetivação: Relato de uma experiência. Physis Revista de Saúde Coletiva, 20(4), 1079-1097. doi:10.1590/S0103-73312010000400002 
Salles, M. M., \& Barros, S. (2007). Reinternação em hospital psiquiátrico: A compreensão do processo saúde/doença na vivência do cotidiano. Revista da Escola de Enfermagem da USP, 41(1), 73-81. doi:10.1590/S008062342007000100010

Venturini, E. (2010). A desinstitucionalização: limites e possibilidades. Revista Brasileira de Crescimento e Desenvolvimento Humano, 20(1), 138-151. Recuperado de http://pepsic.bvsalud.org/pdf/rbcdh/v20n1/18.pdf.

Zambenedetti, G., \& Silva, R. A. N. (2008). A noção de rede nas reformas sanitária e psiquiátrica no Brasil. Psicologia em Revista, 14(1), 131-150. Recuperado

http://www1.pucminas.br/imagedb/documento/DOC_DSC_NOME_ARQUI20 081029093450.pdf.

\section{Sobre os autores}

Gabriela Lemos de Pinho Zanardo é psicóloga pela Pontifícia Universidade Católica do Rio Grande do Sul (PUCRS), residente especialista em Saúde Mental pelo Hospital de Clínicas de Porto Alegre e mestra em Psicologia pela PUCRS. Atualmente é doutoranda do Grupo de Pesquisa Psicologia, Saúde e Comunidades no PPG- Psicologia da PUCRS e bolsista pela Coordenação de Aperfeiçoamento de Pessoal de Nível Superior (CAPES).

gabrielazanardo@hotmail.com

Desirée Luzardo Cardozo Bianchessi é psicóloga pela Pontifícia Universidade Católica do Rio Grande do Sul (PUCRS), especialista em Psicologia Hospitalar e Psicologia Organizacional e do Trabalho, mestra em Psicologia Social e Institucional pela Universidade Federal do Rio Grande do Sul (UFRGS). Atualmente é psicóloga do Hospital de Clínicas de Porto. dbianchessi@hcpa.edu.br

Kátia Bones Rocha é psicóloga pela Pontifícia Universidade Católica do Rio Grande do Sul (PUCRS), mestra em Psicologia pela PUCRS e doutora em Psicologia Clinica i de la Salut pela Universitat Autònoma de Barcelona. Atualmente é professora do Programa de Pós-Graduação e da Faculdade de Psicologia da PUCRS e coordenadora do Grupo de Pesquisa Psicologia, Saúde e Comunidades (PSC). katia.rocha@pucrs.br

Certificamos que todos os autores participaram suficientemente do trabalho para tornar pública sua responsabilidade pelo conteúdo. Gabriela Lemos de Pinho Zanardo e Desireé Luzardo Cardozo Bianchessi contribuíram para a conceitualização, investigação, redação do artigo, revisão crítica, edição e aprovação da versão a ser publicada. Kátia Bones Rocha contribuiu para a redação do artigo, revisão crítica, edição e aprovação da versão a ser publicada. 
Recebido em: 17/01/2017

Revisado em: 20/06/2017

Aceito em: 27/07/2017 